\title{
Activity, Participation, and Goal Awareness After Acquired Brain Injury: A Prospective Observational Study of Inpatient Rehabilitation
}

\author{
Zoe Adey-Wakeling, PhD ${ }^{1,2}$, Laura Jolliffe, $\mathrm{PhD}^{3,4}$, Elizabeth $\mathrm{O}^{\prime}$ Shannessy, BOccTher ${ }^{3}$, Peter Hunter, $\mathrm{MBBS}^{3}$, \\ Jacqui Morarty, $\mathrm{MOT}^{3}$, Ian D. Cameron, $\mathrm{PhD}^{5}$, Enwu Liu, $\mathrm{PhD}^{1,6}$, Natasha A. Lannin, $\mathrm{PhD}^{3,4,7}$ \\ ${ }^{1}$ Department of Rehabilitation, Flinders University of South Australia, Bedford Park, SA; \\ ${ }^{2}$ Department of Rehabilitation, Flinders Medical Centre, Flinders Drive, Bedford Park, SA; \\ ${ }^{3}$ Alfred Health, Melbourne; \\ ${ }^{4}$ Department of Neurosciences, Monash University, Melbourne; \\ ${ }^{5}$ John Walsh Centre for Rehabilitation Research, University of Sydney, Sydney; \\ ${ }^{6}$ Mary MacKillop Institute for Health Research, Australian Catholic University, Melbourne; \\ ${ }^{7}$ College of Science, Health and Engineering, La Trobe University, Melbourne, Australia
}

\begin{abstract}
Objective To examine the frequency and timing of inpatient engagement in meaningful activities within rehabilitation (within and outside of structured therapy times) and determine the associations between activity type, goal awareness, and patient affect.

Methods This prospective observational study performed behavioral mapping in a 42-bed inpatient brain injury rehabilitation unit by recording patient activity every 15 minutes (total 42 hours). The participants were randomly selected rehabilitation inpatients with acquired brain injury; all completed the study. The main outcome measures included patient demographics, observation of activity, participation, goal awareness, and affect.

Results The inpatients spent $61 \%$ of the therapeutic day (8:30 to 16:30) in their single room and were alone $49 \%$ of the time. They were physically socially inactive for $76 \%$ and $74 \%$ of their awake time, respectively, with neutral affect observed for about half of this time. Goal-related activities were recorded for only $25 \%$ of the inpatients' awake time. The odds of physical activity were 10.3-fold higher among in patients receiving support to address their goals within their rehabilitation program (odds ratio=10.3; 95\% confidence interval, 5.02-21.16).

Conclusion Inpatients in a mixed brain injury rehabilitation unit spent a large amount of their awake hours inactive and only participated in goal-related activities for a quarter of their awake time. Rehabilitation models that increase opportunities for physical, cognitive, and social activities outside of allied health sessions are recommended to increase overall activity levels during inpatient rehabilitation.
\end{abstract}

Keywords Rehabilitation, Environment, Brain injury, Activity analysis, Multidisciplinary

Received February 18, 2021; Revised April 11, 2021; Accepted November 8, 2021; Published online December 31, 2021

Corresponding author: Zoe Adey-Wakeling

Department of Rehabilitation, Flinders Medical Centre Level 4, Rehabilitation \& Palliative Care Building Flinders Drive, Bedford Park, SA 5042, Australia. Tel: +61-407-374-260, Fax: +614-8-404-2249, E-mail: zoe.adey-wakeling@sa.gov.au

ORCID: Zoe Adey-Wakeling (https://orcid.org/0000-0001-9231-5250); Laura Jolliffe (https://orcid.org/0000-0002-1369-9442); Elizabeth O'Shannessy (https://orcid.org/0000-0002-9946-853X); Peter Hunter (https://orcid.org/0000-0002-6900-6019); Jacqui Morarty (https://orcid.org/0000-0003-34755551); Ian D. Cameron (https://orcid.org/0000-0003-0147-047X); Enwu Liu (https://orcid.org/0000-0003-2580-3523); Natasha A Lannin (https://orcid. org/0000-0002-2066-8345).

(c) This is an open-access article distributed under the terms of the Creative Commons Attribution Non-Commercial License (http://creativecommons.org/ licenses/by-nc/4.0) which permits unrestricted noncommercial use, distribution, and reproduction in any medium, provided the original work is properly cited. Copyright $\odot 2021$ by Korean Academy of Rehabilitation Medicine 


\section{INTRODUCTION}

Acquired brain injury (ABI) is a major public health issue and a leading cause of disability [1]. Clinical practice guidelines emphasize the importance of goal-directed rehabilitation and maximizing the amount of active therapy to address set goals [2,3]. The Royal Australian College of Physician Standards for Provision of Inpatient Adult Rehabilitation Medicine Services recommend that the appropriate amount of therapy that patients receive will range from a minimum of 3 hours for patients who have the capacity to tolerate this amount of therapy, down to lesser amounts, based on patient need and capacity to participate [4]. Despite these recommendations, inpatient rehabilitation units have historically struggled to meet activity targets. In addition to structured therapy time, engagement in meaningful activities is also an important aspect of patient recovery and well-being. The National Institute for Health and Care Excellence (NICE) Quality Statement defines meaningful activity as including physical, social, and leisure activities that are tailored to the person's needs and preferences [5]. Therefore, complete activity content cannot be considered as structured therapy time alone, as there are opportunities for engagement across all waking hours.

A 2012 review of hospitalized stroke patients [6] found that they spent most of their day inactive (median $48.1 \%$ ), alone (median 53.7\%), and in their bedroom (median $56.5 \%)$. Similarly, a 2004 study [7] observed that stroke patients spent $>50 \%$ of the therapeutic day in bed, $28 \%$ sitting out of bed, and $>60 \%$ alone. Inactivity is not unique to stroke patients; the literature [8] also indicates that hospitalized general medical patients spend $88 \%$ of their therapeutic day in their rooms, $25 \%$ doing nothing, and an additional $17 \%$ watching television. A $2014 \mathrm{ob}-$ servational study [9] with behavior mapping of a 20-bed mixed rehabilitation unit sought to determine whether rehabilitation had improved over the previous decade and to understand if levels of inactivity differed between weekdays and weekends, and reported that rehabilitation inpatients remain inactive and alone, and were $5 \%$ less physically active on weekends [9]

While all these studies have reported low levels of physical and cognitive activity in rehabilitation settings [6-9] it remains unknown whether these findings are comparable in the younger, brain injury population, where patient goals are likely to differ. Additionally, the relationships between patient activity in rehabilitation, goals set, and patient affect have not yet been investigated. The goals pursued in rehabilitation and the levels of patient involvement and empowerment influence rehabilitation outcomes [10]. Therefore, this study aimed to explore how frequently ABI rehabilitation inpatients engaged (participated) in meaningful activities, whether there was a difference in participation in activity levels on weekdays versus weekends, and whether there was a relationship between levels of activity, goal engagement, and patient affect.

\section{MATERIALS AND METHODS}

\section{Study design}

This prospective observational study was conducted using behavioral mapping techniques. To obtain an estimate of patient activity, behavior was audited at 15-minute intervals over 34 consecutive hours (capturing two weekdays, commencing at 7:00 on day 1 and concluding at 17:00 on day 2). Weekend days were also observed for 4 hours each day (a total of 8 hours of weekend observations). This produced 42 hours of observed behavior and $>1,500$ observation points across all participants. The study received institutional ethics approval from Alfred Health (Alfred 355/14). All procedures were performed in accordance with ethical standards, including written informed consent.

\section{Study participants}

The study sample included nine rehabilitation patients with non-progressive acquired brain impairments who were inpatients in a statewide brain injury unit, selected at random using Excel program. All inpatients with a diagnosis of non-progressive acquired injury at the time of auditing were eligible for inclusion. Patients in a posttraumatic amnesic or minimally conscious state were excluded from this study.

The rehabilitation unit environment included singlepatient rooms (each with its own ensuite), therapy spaces and gym, communal dining spaces, secure outdoor areas, and family lounge areas within the ward. The ensuite bathrooms were not observed for privacy reasons; however, patient self-reports of activities undertaken within that space were recorded. For all other spaces, observa- 
tion data were recorded without interference from the patients under observation.

\section{Procedures and observational measures}

Behavioral mapping [11] was used to observe patient participation in meaningful activities and record by location and time. Meaningful activity included "physical, social, and leisure activities that are tailored to a person's needs and preferences [5]. Inactivity was, therefore, defined as the absence of any of these activities. Participation is conceptually defined as "involvement in a life situation [12]. In this study, the operational definition of participation included patient attendance and involvement in a given activity [13]. Time was broadly categorized into that in which therapy was routinely delivered (referred to as the therapeutic day, between 8:30 and 16:30) and non-therapy time, which was defined as the hours outside of hours of the therapeutic day. Awake time was recorded by direct observation of the patient being awake, rather than a defined period, and included both day and night times, which were aggregated. Data were collected by five independent observers over a consecutive 34-hour observation period, working in shifts of 5-8 hours before rotating. A single observer completed the observations on weekend days. The collected data included baseline demographics and observation of activity type (physical, cognitive, and social), participation in activity type, whether participation was directed toward patient-stated goals, and patient affect.

The observation record sheet listed options under their respective domains; for example, under the domain "location, a list of nine locations was provided, including ward space, gym area, patient's room, and other. The observational data were recorded as binary yes/no. In this location domain example, a patient observed in the gym area would be marked as yes on the audit datasheet. The observational recordings included the domains and options of:

- Location (own room, ward space, day room, therapy/ gym, dining, amenities, outside within grounds, outside external to grounds, external leave, other/medical)

- People present (alone, other patient, visitors, allied health, nursing, medical, patient support, etc.)

- Clothing (pyjamas, day clothes, no clothes) and footwear (barefoot, socks, slippers, sports shoes, etc.)
- Awake activity (physical, cognitive, social) or asleep

- Equipment (aids, leisure)

- Goad-directed participation; actively and intentionally working on rehabilitation goal(s)?

- Observed affect (Affect Rating Scale [ARS])

Affect relates to the patient's experience of feeling, emotion, or mood, and was recorded using the modified Philadelphia Geriatric Centre positive and negative ARS [14]. This validated and reliable observational instrument is used to measure positive and negative affects [9]. Consistent with prior audit studies, the ARS was adapted to include a "neutral affect category in addition to the original three positive descriptors (pleasure, interest, contentment) and three negative descriptors (anger, anxiety, sadness) [15].

Observations were carried out every 15 minutes by dedicated research assistants and electronically documented following each observation period. If the patient was not located, not observed was recorded.

\section{Statistical analysis}

The baseline characteristics of the observed inpatients are described without the use of statistics, given the small sample size. A total of 1,550 observations was made across all nine inpatients (range, 164-180 observations per patient). Multivariate binary logistic analysis using generalized estimating equation (GEE) models were used to investigate the associations between the considered exposure and outcome variables of the repeated measurements. The outcome variables for the study were binary variables (activity, yes or no). For each potential association, a GEE model was used to calculate the odds ratios (ORs) with logit link function and exchangeable correlation structure controlling for age, sex, injury type, and mobility at the time of the audit. Statistical significance was set at a two-sided p-value of $<0.05$. Analyses were performed using SAS version 9.3 (SAS Institute Inc., Cary, NC, USA) [16].

\section{RESULTS}

Nine inpatients (33\% female) aged $25-59$ years (mean, 40 years) participated in the study. Five participants were diagnosed with non-traumatic brain hemorrhage (one with a dual diagnosis of encephalitis), and four were diagnosed with traumatic brain injury. Two participants 
walked unaided at the time of the audit, two used a wheeled walking frame, four used a wheelchair independently, and one was non-ambulant (required attendantpropelled wheelchair). Table 1 compares the participants' characteristics to all inpatients admitted to the same ward over the preceding 12 months for reference.

Over the 42 hours of observation, a minimum of 164 individual observations per participant were recorded (median number of observations, 171), producing a total of 1,550 data points.

Between 08:30 and 16:30 (therapeutic day), patients spent $29 \%$ of their time with allied health therapists, $62 \%$ of the time in their single rooms, and $49 \%$ of their time alone. These proportions were very similar among patient locations during awake time and on both weekdays and weekend days. Patients were physically and socially inactive $76 \%$ and $74 \%$ of their awake time (i.e., excluding sleep time), respectively. Organized activities such as communal dining and group therapy programs increased both physical and social activity levels in patients who participated. Patients were only actively participating in activities related to their rehabilitation goals for $25 \%$ of their awake time and $36 \%$ of the therapeutic day, with $80 \%$ of the time spent by an allied health therapist on goal-related activities.

The correlations between activity type, participation, and people present, activity location, and day of the week (weekend versus weekday) are shown in Table 2. The presence of visitors and nursing staff significantly increased the odds of participating in physical and social activities, as observed from the role they played for some participants in supporting self-practice therapy programs prescribed by allied health therapists.

\section{Physical activity levels}

The proportion of time spent engaged in physical activity did not differ between weekend and weekdays (OR=1.92; 95\% confidence interval [CI], 0.88-4.19; $\mathrm{p}=0.1002$ ). Being alone was associated with less physical activity (OR=0.09; 95\% CI, 0.05-0.17; $<<0.0001$ ), while the presence of a nurse (OR=3.92; 95\% CI, 2.54-6.05; $\mathrm{p}<0.0001)$, visitor (OR=5.87; 95\% CI, 2.21-15.5; $\mathrm{p}<0.0001)$, allied health therapist $(\mathrm{OR}=15.3 ; 95 \% \mathrm{CI}, 9.06-25.73$; $\mathrm{p}<0.0001)$, or patient support (OR=4.04; 95\% CI, 1.22 13.37; $\mathrm{p}=0.0224$ ) was associated with a higher probability of engaging in a physical activity task. In contrast, the presence of rehabilitation physicians was not associated with any changes in patient participation $(\mathrm{OR}=0.58$; $95 \%$

Table 1. Characteristics of the participants compared to the ward population

\begin{tabular}{lcc}
\hline \multicolumn{1}{c}{ Characteristic } & $\begin{array}{c}\text { Study participants } \\
(\mathbf{n}=\mathbf{9})\end{array}$ & $\begin{array}{c}\text { Ward population (2015-2016) } \\
(\mathbf{n}=\mathbf{1 9 0})\end{array}$ \\
\hline Age (yr) & $40 \pm 10$ & $44 \pm 17$ \\
Sex, male & $6(67)$ & $134(71)$ \\
Admission FIM motor score & $27.4 \pm 24.7$ & $41.9 \pm 25.9$ \\
Admission FIM cognition score & $15.3 \pm 8.9$ & $14.3 \pm 8.4$ \\
Acquired brain injury type & & \\
TBI & $3(33)$ & $139(73)$ \\
Non-traumatic hemorrhage & $5(56)$ & $72(38)$ \\
Other & $1(11)$ & $46(24)$ \\
ABI severity) (lowest GCS) & - & 84.6 \\
Time from injury to first observation in weeks & $25(37)^{\mathrm{b})}$ & - \\
Time from rehabilitation admission to first observation (wk) & $14 \pm 10$ & - \\
Behavioral support plan (number with plan) & $6(67)$ & - \\
Mobility status at the time of audit (FIM walk item) & $5.3(1.8)$ & - \\
Compensation status (number compensable) & $1(11)$ & $56(29)$ \\
\hline
\end{tabular}

Values are presented as mean \pm standard deviation or number (\%).

FIM, Functional Independence Measure; TBI, traumatic brain injury; ABI, acquired brain injury; GCS, Glasgow Coma Scale.

${ }^{\text {a) }}$ Patients with TBI, ${ }^{\text {b) }}$ the number in parentheses indicates interquartile range. 


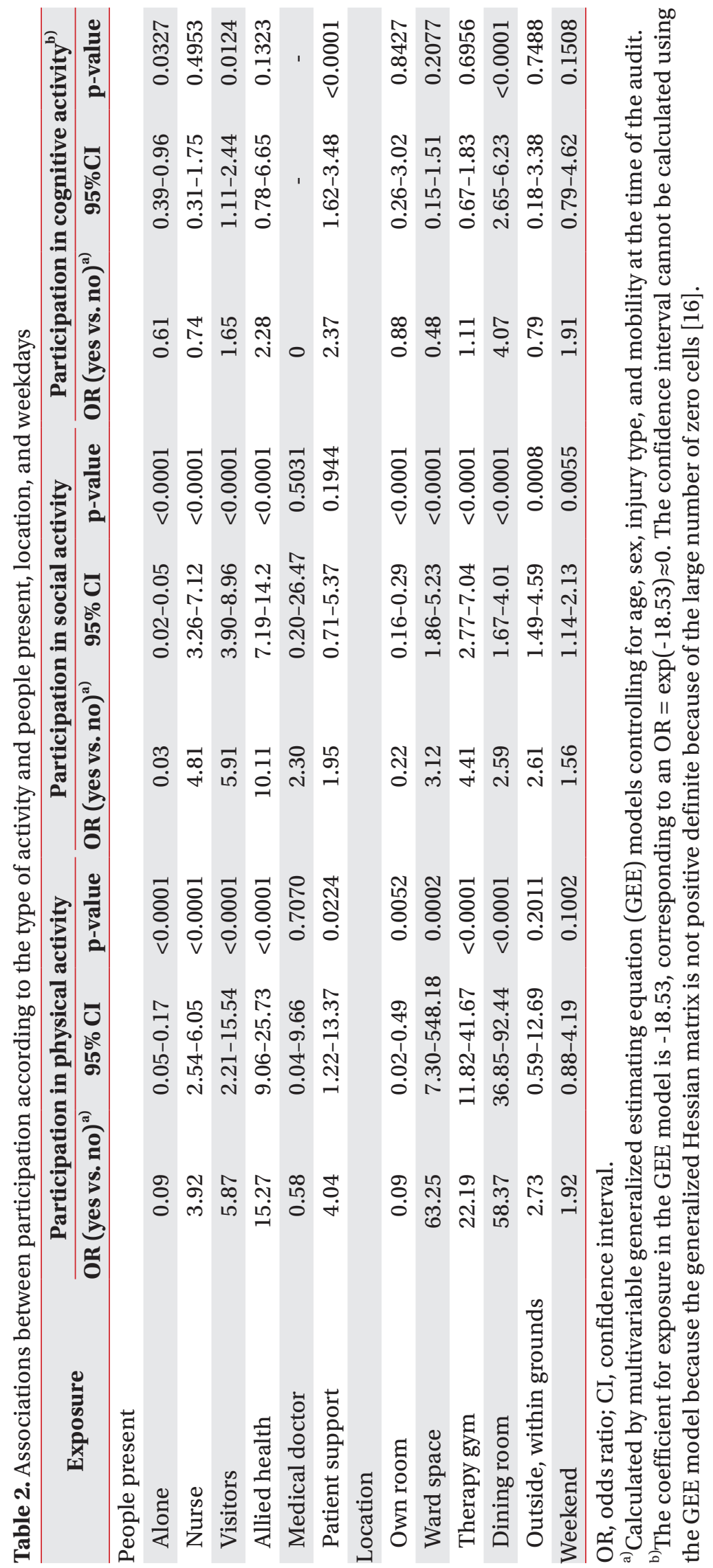


CI, 0.05-6.68; $\mathrm{p}=0.6653)$. Patient location appeared to influence physical activity levels. Patient time spent in the ward space (OR=63.25; 95\% CI, 7.30-548.18; $\mathrm{p}=0.0002)$, therapy gym $(\mathrm{OR}=22.19 ; 95 \% \mathrm{CI}, 11.82-41.67$; $<<0.0001)$, or the dining room $(\mathrm{OR}=58.37 ; 95 \% \mathrm{CI}, 36.85-92.44$; $\mathrm{p}<0.0001$ ) was associated with a higher probability of physical activity, while patient time spent in their room was associated with a higher probability of less physical activity (OR=0.09; 95\% CI, 0.02-0.49; p=0.0052). Surprisingly, patients outside but within the hospital grounds were not associated with higher physical activity levels (OR=2.73; 95\% CI, 0.59-12.69; $\mathrm{p}=0.2011$ ).

\section{Social activity levels}

We investigated patient contact with others to understand its effects on social activity levels. As predicted, being alone was associated with less social activity (OR=0.03; 95\% CI, 0.02-0.05; $\mathrm{p}<0.0001$ ), while the presence of visitors (OR=5.91; 95\% CI, 3.90-8.96; $\mathrm{p}<0.0001)$ or a nurse $(\mathrm{OR}=4.81 ; 95 \% \mathrm{CI}, 3.26-7.12 ; \mathrm{p}<0.0001)$ were associated with higher probabilities of social activity. The presence of neither rehabilitation physicians ( $O R=2.3 ; 95 \% \mathrm{CI}, 0.2-26.47 ; \mathrm{p}=0.5031$ ) nor patient support (OR=1.95; 95\% CI, 0.71-537; $\mathrm{p}=0.1944)$ increased the level of social activity. Similar to the amount of physical activity, patient time spent in the ward space $(\mathrm{OR}=3.12$; 95\% CI, 1.86-5.23; $\mathrm{p}<0.0001)$, therapy gym (OR=4.41; 95\% CI, 2.77-7.04; $\mathrm{p}<0.0001)$, dining room (OR=2.59; 95\% CI, $1.67-4.01 ; \mathrm{p}<0.0001)$, and outside within the grounds $(\mathrm{OR}=2.61 ; 95 \% \mathrm{CI}, 1.49-4.59 ; \mathrm{p}=0.0008)$ was associated with a higher probability of social activity, while patient time spent in their room was associated with less social activity (OR=0.22; 95\% CI, 0.16-0.29; p<0.0001). Unlike our findings for physical activity, we observed a higher probability of participating in a social activity task on weekend versus weekdays (OR=1.56; 95\% CI, 1.14-2.13; $\mathrm{p}=0.0055$ ).

\section{Cognitive activity levels}

Patient time spent alone (OR=0.61; 95\% CI, 0.39-0.96; $\mathrm{p}=0.0327$ ) was associated with less cognitive activity. The presence of visitors $(\mathrm{OR}=1.65 ; 95 \% \mathrm{CI}, 1.11-2.44$; $\mathrm{p}=0.0124)$ and time spent in dining rooms $(\mathrm{OR}=4.07$; 95\% CI, 2.65-6.23; $\mathrm{p}<0.0001$ ) were associated with higher probabilities of participating in a cognitive activity.

Participation in any activity was greatest when the patient was in the presence of a nurse, visitors, or allied health, and outside of their room. The highest probability of goal-directed participation was observed in the therapy gym and the presence of allied health. Conversely, being alone or in their room was associated with less participation in any activity. Overall, physical (OR=10.3; 95\% CI, 5.02-21.16) and social (OR=5.17; 95\% CI, 2.63-10.17) activity levels were higher in those patients who were observed to participate in activities related to the goals within their rehabilitation programs (Table 3).

Recording and mapping of patient affect showed that an overall neutral affect was observed $47 \%$ of the time, with pleasure, interest, or contentment only evident $35 \%$ of the total time. These ratings were comparable between the total awake and therapy time observations (Table 4).

Table 3. Odds ratios of goal activity per outcome variable

\begin{tabular}{lccc}
\hline \multirow{2}{*}{ Outcome variable } & \multicolumn{3}{c}{ Goal-directed participation } \\
\cline { 2 - 4 } & $\mathbf{O R}^{\text {a) }}$ (yes vs. no) & $\mathbf{9 5 \%} \mathbf{C I}$ & p-value \\
\hline Therapeutic hours & 5.34 & $2.57-11.08$ & $<0.0001$ \\
All physical activity & 10.30 & $5.02-21.16$ & $<0.0001$ \\
All cognitive activity & 2.74 & $1.21-6.22$ & 0.016 \\
All social activity & 5.17 & $2.63-10.17$ & $<0.0001$ \\
\hline
\end{tabular}

OR, odds ratio; $\mathrm{CI}$, confidence interval.

OR and p-values were calculated by four generalized estimating equation (GEE) models. The outcome variables were therapeutic hours, all physical activity, all cognitive activity, and all social activity. For each GEE model, the covariates were goal-directed participation, age, sex, injury type, and mobility at the time of audit.

${ }^{a}$ For patients actively working on a goal-related activity, the OR of the activity per outcome variable was compared to patients not working on a goal-related activity. 
Table 4. Observed affects during awake time and therapeutic day

\begin{tabular}{|c|c|c|c|}
\hline \multirow{2}{*}{\multicolumn{2}{|c|}{ Observed affect (experience of feeling, emotion, or mood) }} & \multicolumn{2}{|c|}{ Recorded observations } \\
\hline & & \multirow{2}{*}{$\begin{array}{c}\begin{array}{c}\text { Awake time } \\
(\mathbf{n}=\mathbf{9 2 1})\end{array} \\
64(7)\end{array}$} & \multirow{2}{*}{$\begin{array}{c}\text { Therapeutic day } \\
\text { (n=927) }\end{array}$} \\
\hline Pleasure & $\begin{array}{l}\text { Smiling, laughing, stroking, nodding, singing, arm or hand } \\
\text { outstretched, open arm gesture }\end{array}$ & & \\
\hline Anger & $\begin{array}{l}\text { Clenched teeth, grimace, shouting, berating, physical ag- } \\
\text { gression }\end{array}$ & $0(0)$ & $0(0)$ \\
\hline Anxiety & $\begin{array}{l}\text { Furrowed brow, motor restlessness, repeated agitated mo- } \\
\text { tions, facial expressions of fear or worry, sigh, withdrawal } \\
\text { from others tremor, tight facial muscles, calling repeti- } \\
\text { tively }\end{array}$ & $28(3)$ & $28(3)$ \\
\hline Neutral & No observable affect & $497(54)$ & $436(47)$ \\
\hline Depression/sadness & Crying/tears, moaning, mouth turned down & $0(0)$ & $0(0)$ \\
\hline Interest & $\begin{array}{l}\text { Eyes following object, intent fixation on object or person, } \\
\text { visual scanning, facial motor or verbal feedback, eye con- } \\
\text { tact maintained, body or vocal response to music, gaze, } \\
\text { body turning or movement towards a person or object on } \\
\text { interest. }\end{array}$ & $193(21)$ & $158(17)$ \\
\hline Contentment & $\begin{array}{l}\text { Comfortable posture, sitting or lying down, smooth facial } \\
\text { muscles, lack of tension in limbs or neck, slow move- } \\
\text { ments, relaxed body stance, lightening of frown or worry } \\
\text { line }\end{array}$ & $120(13)$ & $112(12)$ \\
\hline
\end{tabular}

Values are presented as number (\%).

\section{DISCUSSION}

Consistent with the stroke literature, a comparative sample of inpatients in the ABI rehabilitation ward spent most of the day inactive and alone in their rooms. While this finding is not new, it is interesting to note that this significantly younger ABI sample (mean age, 40 years) did not demonstrate increased overall activity levels compared to those in the older stroke rehabilitation cohort (mean age, 78 years) observed in previous literature [9]. This study additionally correlated spaces and presence of staff, noting that participation was greatest in presence of a nurse, visitor, or allied health team member, and when the participants were outside of their rooms. Observations of solitude and inactivity were consistent between hours within and outside of the therapeutic day, as well as weekdays and weekend days, suggesting that higher staffing levels alone (weekdays during the therapeutic day) did not appear to increase patient engagement levels. These novel and important findings could be used to improve patient activity, with directed staff efforts focused on individualized activity targets.

Nurses could be targeted as leaders of initiatives to increase patient activity and participation within rehabilitation units. Nursing staff are in a prime position to practice learned therapy strategies, embed strategies into patients' daily routines, and encourage patient independence. Theofanis [17] described the importance of nursing engagement beyond the standard paradigm of routine interventions, encouraging a focus on "the wider essential care aspects to enable faster and more complete recovery. This study echoes earlier research [18] reporting the value of nursing input on brain injury recovery. We also found limited patient engagement in activities outside of regular therapeutic hours. Consistent with previous studies on rehabilitation [7], these findings suggested the need for changes at an organizational level to increase levels of participation in rehabilitation. The engagement of nursing staff in co-treatment sessions with allied health, working parties, and post-graduate and point-of-care education strategies may be essential for increasing inpatient activity towards clinical guideline recommendations.

Our findings emphasize the importance of rehabilitation. Social activity was higher when the patients were involved in communal dining, while physical activity was 
higher when visitors were involved in the rehabilitation programs and nursing staff carried over therapy programs outside of therapy sessions. Social activity levels were also higher when the participants were outside, indicating that the entire rehabilitation environment should be considered when seeking opportunities to increase activity levels. Strategies such as increasing environmental opportunities for independent or semi-supervised patient practice may also be valuable. An accessible outdoor garden, kitchen, independent practice gym, and welcoming spaces for socializing, as well as providing family skill training and education may allow patients to participate in rehabilitation activities outside of therapeutic hours. Around-the-clock engagement and encouragement from within a service may also impact patient affect. The patients in the present study rarely displayed a positive effect: reduced activity levels may have influenced their affect, which may have further decreased their motivation to participate. Thus, changing service delivery may have far-reaching consequences. Increasing accessible and appropriately goal-focused activities should be a target during inpatient rehabilitation for patients of all ages. Our findings reinforce the importance of the whole environment, as well as strategies such as communal dining and family engagement. The regular provision of practice tasks by allied health may encourage increased patient activity outside of therapeutic day; however, this passive activity should be coupled with education and encouragement and allied health should be optimally placed to ensure that family and caregivers can facilitate afterhours therapy. The findings revealed the lowest activity levels in the presence of medical staff, highlighting the lack of physician involvement in promoting engagement. Medical focus on patient activity is crucial and suggests an opportunity to coordinate and plan active and personcentered interventions. While around-the-clock nursing shifts provide regular occasions for nurses to play a pivotal role in increasing activity out of hours, all clinicians must seek to change the culture to increase opportunities for additional therapy.

This study had several limitations. While this study observed only nine inpatients, the large number of continuous observations $(>1,500)$ increased the robustness of the findings. The repeated-measures methodology simultaneously increased the statistical power and reduced the costs of the study [19]. However, the observational design led to inherent sources of error. With overt observation, it is plausible that both clinicians and patients could alter their behaviors. We do not believe this to have been the case, as previous audit studies have reported similar results. To mitigate this potential Hawthorne effect, we used a large number of continuous observations and a 34-hour continuous observation period; however, this potential limitation must be acknowledged.

In conclusion, while a substantial body of knowledge exists regarding how much rehabilitation is needed to improve outcomes [20], audit results indicate that clinicians continue to struggle to meet these clinical practice guidelines. The findings of this study demonstrate the need for enhanced engagement in meaningful activities outside of prescribed allied health hours. An increased focus on enhancing opportunities for engagement in meaningful activities is required for rehabilitation. The findings of this study also underscore the need for a collective effort to implement models of rehabilitation that maximize physical, cognitive, and social activities to enhance meaningful participation after ABI.

\section{CONFLICT OF INTEREST}

No potential conflict of interest relevant to this article was reported.

\section{ACKNOWLEDGMENTS}

This project was funded by WorkSafe Victoria and the Transport Accident Commission through the Institute for Safety, Compensation and Recovery Research (ISCRR) (No. GNT108). The following authors were supported by fellowships: Laura Joliffe (NHMRC, No. GNT1114522) and Natasha A. Lannin (National Heart Foundation of Australia, Future Leader Fellowship, No. GNT102055).

\section{AUTHOR CONTRIBUTION}

Conceptualization: Lannin NA, Hunter P, Morarty J, Cameron ID. Methodology: Lannin NA, Hunter P, Morarty J, Cameron ID. Data collection: Joliffe L, O'Shannessy E, Lannin NA. Formal analysis: EL, Adey-Wakeling Z. Funding acquisition: Lannin NA. Writing - original draft: Adey-Wakeling Z, O'Shannessy E, Lannin NA. Writing - review and editing: Adey-Wakeling Z, O'Shannessy E, 
Lannin NA. Approval of final manuscript: all authors.

\section{REFERENCES}

1. Australian Institute of Health and Welfare. Disability in Australia: acquired brain injury [Internet]. Canberra, Australia: Australian Institute of Health and Welfare; 2007 [cited 2021 Dec 15]. Available from: https:// www.aihw.gov.au/getmedia/1f719b27-6b93-434ab0e6-997b4ead061a/bulletin55.pdf.aspx?inline=true.

2. Royal College of Physicians, Intercollegiate Stroke Working Party. National clinical guidelines for stroke, 5th edition [Internet]. London, UK: Royal College of Physicians; 2016 [cited 2021 Dec 15]. Available from: https://www.strokeaudit.org/Guideline/Full-Guideline.aspx.

3. Stroke Foundation. Clinical guidelines for stroke management [Internet]. Melbourne, Australia: Stroke Foundation; 2017 [cited 2021 Dec 15]. Available from: https://informme.org.au/Guidelines/Clinical-Guidelines-for-Stroke-Management-2017.

4. Royal Australasian College of Physician. Standards for the provision of inpatient adult rehabilitation medicine services in public and private hospitals 2011 [Internet]. Sydney, Australia: Royal Australasian College of Physician; 2012 [cited 2021 Dec 15]. Available from: https://www.racp.edu.au/docs/default-source/policy-and-adv/afrm/afrm-standards-provision-of-inpatient-adult-rehabilitation-medicine-services-2011. pdf?sfvrsn=564f2dla_6.

5. National Institute for Health and Care Excellence. Mental wellbeing of older people in care homes Quality standard [QS50] - Quality statement 1: Participation in meaningful activity [Internet]. London, UK: National Institute for Health and Care Excellence; 2013 [cited 2021 Dec 15]. Available from: https:// www.nice.org.uk/guidance/qs50/chapter/qualitystatement-1-participation-in-meaningful-activity.

6. West T, Bernhardt J. Physical activity in hospitalised stroke patients. Stroke Res Treat 2012;2012:813765.

7. Bernhardt J, Dewey H, Thrift A, Donnan G. Inactive and alone: physical activity within the first 14 days of acute stroke unit care. Stroke 2004;35:1005-9.

8. Kuys SS, Dolecka UE, Guard A. Activity level of hospi- tal medical inpatients: an observational study. Arch Gerontol Geriatr 2012;55:417-21.

9. Janssen H, Ada L, Bernhardt J, McElduff P, Pollack M, Nilsson M, et al. Physical, cognitive and social activity levels of stroke patients undergoing rehabilitation within a mixed rehabilitation unit. Clin Rehabil 2014;28:91-101.

10. Holmqvist LW, von Koch L. Environmental factors in stroke rehabilitation. BMJ 2001;322:1501-2.

11. Ng CF. Behavioral mapping and tracking. In: Gifford R, editor. Research methods for environmental psychology. Malden, MA: Wiley-Blackwell; 2016. p. 29-52.

12. World Health Organization. International Classification of Functioning, Disability and Health: short version. Geneva, Switzerland: World Health Organization; 2001.

13. Imms C, Adair B, Keen D, Ullenhag A, Rosenbaum P, Granlund M. 'Participation': a systematic review of language, definitions, and constructs used in intervention research with children with disabilities. Dev Med Child Neurol 2016;58:29-38.

14. Watson D, Clark LA, Tellegen A. Development and validation of brief measures of positive and negative affect: the PANAS scales. J Pers Soc Psychol 1988;54:1063-70.

15. Lawton MP, Van Haitsma K, Klapper J. Observed affect in nursing home residents with Alzheimer's disease. J Gerontol B Psychol Sci Soc Sci 1996;51:P3-14.

16. Dmitrienko A. Analysis of clinical trials using SAS: a practical guide. Cary, NC: SAS Institute; 2005.

17. Theofanidis D. Nursing interventions and rehabilitation activities for stroke patients. J Nurs Care 2016;5:e131.

18. Burton C, Gibbon B. Expanding the role of the stroke nurse: a pragmatic clinical trial. J Adv Nurs 2005;52:640-50.

19. Guo Y, Logan HL, Glueck DH, Muller KE. Selecting a sample size for studies with repeated measures. BMC Med Res Methodol 2013;13:100.

20. Schneider EJ, Lannin NA, Ada L, Schmidt J. Increasing the amount of usual rehabilitation improves activity after stroke: a systematic review. J Physiother 2016;62:182-7. 